\title{
A new generation of high-speed electro-optical transceivers and flexible bandwidth wavelength selective switches for coherent DCI
}

The QAMeleon project approach

Zervos, C.; Spyropoulou, M.; Kanakis, I.; Lazarou, I.; Velthaus, K. O.; Rouvalis, E.; Torfs, G.; Goobar, E.; Santos, R.; Tessema, N. M.

Total number of authors:

20

Published in:

Proceedings of SPIE

Link to article, DOI:

10.1117/12.2509454

Publication date:

2019

Document Version

Publisher's PDF, also known as Version of record

Link back to DTU Orbit

Citation (APA):

Zervos, C., Spyropoulou, M., Kanakis, I., Lazarou, I., Velthaus, K. O., Rouvalis, E., Torfs, G., Goobar, E., Santos, R., Tessema, N. M., Lamprecht, T., Johansen, T. K., Hersent, R., Mardoyan, H., Dris, S., Alexoudi, T., Giardina, P. G., Roccato, D., Vickers, G., \& Avramopoulos, H. (2019). A new generation of high-speed electrooptical transceivers and flexible bandwidth wavelength selective switches for coherent DCl: The QAMeleon project approach. In R. T. Chen, \& H. Schroder (Eds.), Proceedings of SPIE (Vol. 10924). [109240E] SPIE International Society for Optical Engineering. Proceedings of SPIE - The International Society for Optical Engineering Vol. 10924 https://doi.org/10.1117/12.2509454

\section{General rights}

Copyright and moral rights for the publications made accessible in the public portal are retained by the authors and/or other copyright owners and it is a condition of accessing publications that users recognise and abide by the legal requirements associated with these rights.

- Users may download and print one copy of any publication from the public portal for the purpose of private study or research.

- You may not further distribute the material or use it for any profit-making activity or commercial gain

- You may freely distribute the URL identifying the publication in the public portal 


\section{A new generation of high-speed electro-optical transceivers and flexible bandwidth wavelength selective switches for coherent $\mathrm{DCl}$ : the QAMeleon project approach}

C. Zervos, M. Spyropoulou, I. Kanakis, I. Lazarou, K.-O. Velthaus, et al.

C. Zervos, M. Spyropoulou, I. Kanakis, I. Lazarou, K.-O. Velthaus, E.

Rouvalis, G. Torfs, E. Goobar, R. Santos, N. M. Tessema, T. Lamprecht, T. K. Johansen, R. Hersent, H. Mardoyan, S. Dris, T. Alexoudi, P. G. Giardina, D. Roccato, G. Vickers, H. Avramopoulos, "A new generation of high-speed electro-optical transceivers and flexible bandwidth wavelength selective switches for coherent DCI: the QAMeleon project approach," Proc. SPIE 10924, Optical Interconnects XIX, 109240E (4 March 2019); doi: $10.1117 / 12.2509454$

SPIE. Event: SPIE OPTO, 2019, San Francisco, California, United States 


\title{
A new generation of high-speed electro-optical transceivers and flexible bandwidth wavelength selective switches for coherent DCI: The QAMeleon project approach
}

\author{
C. Zervos ${ }^{\mathrm{a}}$, M. Spyropoulou ${ }^{\mathrm{a}}$, I. Kanakis ${ }^{\mathrm{a}}$, I. Lazarou ${ }^{\mathrm{a}}$, K.-O. Velthaus ${ }^{\mathrm{b}}$, E. Rouvalis ${ }^{\mathrm{c}}$, G. Torfs ${ }^{\mathrm{d}}$, \\ E. Goodbar ${ }^{\mathrm{e}}$, R. Santos ${ }^{\mathrm{f}}$, N.M. Tessema ${ }^{\mathrm{g}}$, T. Lamprecht ${ }^{\mathrm{h}}$, T. K. Johansen ${ }^{\mathrm{i}}$, R. Hersent ${ }^{\mathrm{j}}$, H. Mardoyan $^{\mathrm{k}}$,

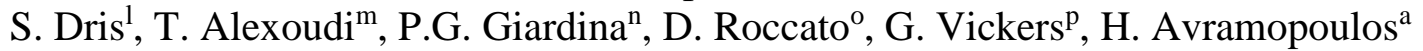 \\ aPhotonics Communications Research Laboratory, National Technical University of Athens, Greece; \\ ${ }^{b}$ Fraunhofer-Institut für Nachrichtentechnik, Heinrich-Hertz-Institut, Berlin, Germany; \\ ${ }^{c}$ Finisar Germany GmbH Reuchlinstrasse 10/11, 10553 Berlin, Germany; \\ ${ }^{\mathrm{d} I D L a b, ~ I N T E C, ~ G h e n t ~ U n i v e r s i t y ~-~ i m e c, ~} 9052$ Ghent, Belgium; \\ ${ }^{e}$ Finisar Sweden AB, Bruttovägen 3, 17543 Järfälla, Sweden;

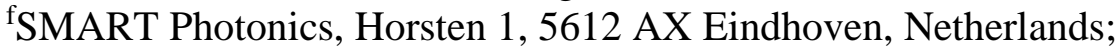 \\ gInstitute of Photonic Integration, Eindhoven University of Technology, Eindhoven, Netherlands; \\ ${ }^{\mathrm{h}}$ Vario-Optics AG, Mittelbissaustr. 7, CH-9410 Heiden, Switzerland; \\ ${ }^{i}$ Department of Electrical Engineering, Technical University of Denmark, Denmark; \\ jIII-V Lab, 1 avenue Augustin Fresnel, 91767 Palaiseau, France; \\ kNokia Bell Labs, Paris-Saclay, 91620 Nozay, France; \\ ${ }^{l}$ VPIphotonics GmbH, 10587 Berlin, Germany; \\ mDepartment of Informatics, Center for Interdisciplinary Research and Innovation, Aristotle \\ University of Thessaloniki, Greece; \\ ${ }^{\mathrm{n} N e x t w o r k s, ~ P i s a, ~ I t a l y ; ~}$ \\ ${ }^{\circ}$ Transport Innovation, Telecom Italia, 10148 Torino, Italy; \\ pOptocap Ltd / ALTER Technology Group TÜV Nord, Scotland, United Kingdom
}

\begin{abstract}
The ever-increasing demands in traffic fueled by bandwidth hungry applications are pushing data centers to their limits challenging the capacity and scalability of currently established transceiver and switching technologies in data center interconnection (DCI) networks. Coherent optics emerged as a promising solution for inter-DCIs offering unprecedented capacities closer to data centers and relaxing the power budget restrictions of the link. QAMeleon, an EU funded R\&D project, is developing a new generation of faster and greener sliceable bandwidth-variable electro-optical transceivers and WSS switches able to handle up to 128 Gbaud optical signals carrying flexible M-QAM constellations and novel modulation techniques. A summary of the progress on the QAMeleon transponder and Reconfigurable Optical Add/Drop Multiplexer (ROADM) concepts is presented in this paper.
\end{abstract}

Keywords: data center interconnection (DCI), bandwidth-variable optical transceivers, wavelength selective switch (WSS), coherent optics, photonic integration, high-speed electronic ICs.

\section{INTRODUCTION}

QAMeleon is aiming to deliver a new generation of photonic devices towards scaling core and metro networks to the next decade. Currently, telecom operators are hard pressed to keep up with the soaring, increasingly volatile traffic that is traversing their networks. New video services are setting the busy-hour internet on a steep growth curve reaching $36 \%$

Optical Interconnects XIX, edited by Henning Schröder, Ray T. Chen, Proc. of SPIE Vol. 10924 109240E · @ 2019 SPIE · CCC code: 0277-786X/19/\$18 · doi: 10.1117/12.2509454 
compound annual growth rate (CAGR), while the average traffic still is estimated at a hefty $25 \% \mathrm{CAGR}^{[1]}$. The operators are forced to plan network capacity according to peak rates rather than average rates thusly wastefully overprovisioning their networks to accommodate these high peak-to-average ratios. The skyrocketing demand has driven equipment manufacturers into a speed race where 200G shipments are now ramping up while the first 600G products have already emerged in $2018^{[15]}$, scaling symbol rates to $64 \mathrm{Gbaud}^{[2]}$. In this regards, component and system vendors are well underway with the development of their 64 Gbaud portfolio to meet these demands, however the enabling technologies to shift to the next gear of 128 Gbaud are urgently being sought. Meanwhile, telecommunication companies must deal with reduced profitability and suppressed margins, as end users demand higher bandwidth and better Quality-of-Service at the same price. This has created an increasingly suffocating model that cannot be escaped from with gradual cost reduction and capacity increases but rather needs innovative concepts that make networks more efficient and dynamic. The benefits of elastic networking are well-established by now ${ }^{[3]}$, whereas SDN is gaining traction, towards point-and-click provisioning of resources and delivery of new services. Although these concepts laid the foundations of programmable optical networks, there is still a long way to go towards fully automated and efficient networking. 'Sliceability' is arguably the next evolutionary step in network programmability since it can bring further reductions in CAPEX and OPEX well exceeding $30 \%{ }^{[4],[5]}$. Sliceable transponders can handle multiple flows and establish point-to-multipoint connections, optimizing hardware resource utilization and extending the use of optical switching over costly and energy-hungry electronic switches. Hence there is a great need for photonic technologies that enable sliceable transponder and ROADMs components, along with the framework that facilitates their programmability in an SDN context.

At the transponder side, QAMeleon is developing transceiver components that enable the switch to 128 Gbaud and will bring significant savings in footprint $(>13 \times)$, energy/bit $(10.4 \times)$ and cost/bit $(>4.3 \times)$. At the ROADM side, QAMeleon is developing large-scale flex-grid wavelength-selective switches $(1 \times 24$ WSS $)$ and transponder aggregators $(8 \times 24$ TPA), with the target of reducing the footprint and the cost/port by more than $40 \%$ and $28 \%$ respectively and achieving energy savings per ROADM node that reach $4 \times$. In order to address the emerging needs of $5 \mathrm{G}$ network backhaul and DCI metroaccess networks where dynamicity is pivotal, QAMeleon is developing an integrated flex-grid $1 \times 4$ WSS with nanosecondscale switching time that is scalable to large channel counts (i.e. full C-band). The $1 \times 4$ WSS will enable the savings in footprint, energy consumption and cost by $20 \times, 11.5 \times$ and $36 \%$ respectively. QAMeleon will also deliver the entire overarching SDN framework for controlling the developed $3 \mathrm{~Tb} / \mathrm{s}$ sliceable bandwidth-variable transponder (S-BVT) and ROADM "white boxes". The developed devices will be validated in scalable lab and field-trial demonstrators.

\section{QAMELEON TRANSPONDER CONCEPT}

Scaling the speed of flexible transceivers stumbles upon the huge disparity between the bandwidth of photonic components and the bandwidth of the interfacing digital electronics, most notably digital analogue converters (DACs). Although photonic components with 3-dB response exceeding $40 \mathrm{GHz}$ are available, complementary metal-oxide semiconductor (CMOS) DACs typically used in transponders are bound to moderate bandwidths well below $30 \mathrm{GHz}$, whereas alternative technologies exhibit limited interest due to their extremely high-power consumption which exceeds the total allowable power consumption of the CFP2 form factor ${ }^{[23]}$. QAMeleon addresses the bandwidth gap between photonics and electronics with an analog signal interleaving concept, presented in Figure 1. This approach allows interleaving the outputs of two high-speed DACs into a very-high-speed analog signal that can be used for feeding the photonic components, thus de-correlating the bandwidth of the DAC with that of the photonics. The same principle is followed at the receiver side, but the other way around: The high-speed analog signal received at the photodetector is de-interleaved into lower bandwidth tributaries, which can be digitized by lower-bandwidth analogue to digital converters (ADCs). 

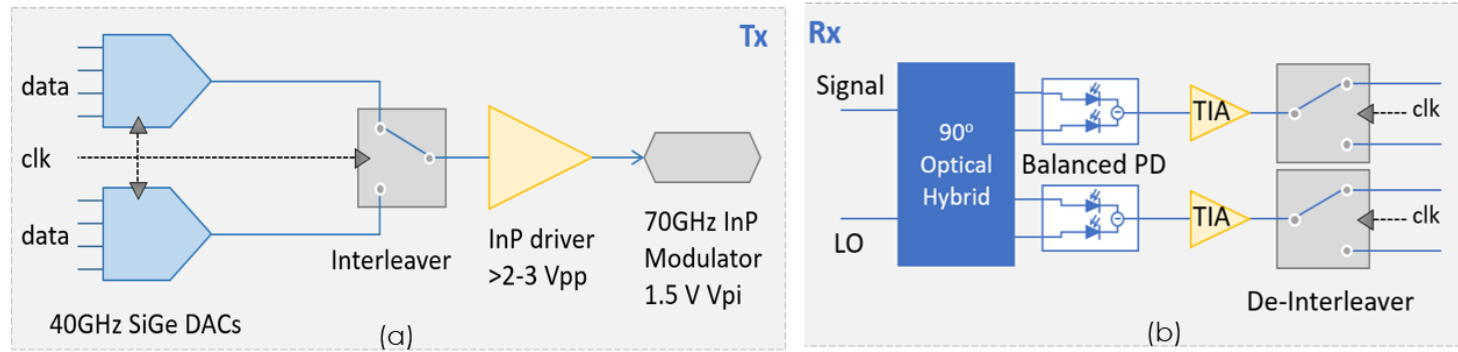

Figure 1: (a,b) QAMeleon transponder bandwidth interleaving concept for scaling speed to 128 Gbaud. (a) The outputs of two SiGe BiCMOS DACs are interleaved to form a high speed 128 Gbaud signal that will drive the $70 \mathrm{GHz}$ InP IQ Mach Zehnder Modulator after linear amplification with an InP-HBT linear driver, (b) The receiver high speed signal is de-interleaved at the receiver into lower bandwidth tributaries.

QAMeleon exploits the best of electronics ICs by combining high resolution Silicon Germanium (SiGe) Bipolar transistor CMOS (BiCMOS) DACs with high bandwidth Indium Phosphide (InP) Heterojunction Bipolar Transistor (HBT) electronics yielding a large effective number of bits (ENOB) that are used for the generation of complex modulation formats rather than for increasing transmitter (Tx) bandwidth. The synergy between SiGe BiCMOS and InP-HBT technologies allows to generate high speed signals in the most power efficient way.

\section{SiGe BiCMOS electronics}

To reduce the power consumption and ensure adequate signal quality, the data generation is split into two parts. First, two lower speed signals of 64 Gbaud will be generated using a 6-bit DAC with an ENOB equal to 5. Next, these two signals are time-interleaved into a single 128 Gbaud signal. To realize the very broadband devices required by QAMeleon, the ST's $55 \mathrm{~nm}$ BiCMOS is chosen. The layout of the first generation 4-to-1 interleaver is shown in Figure 2.

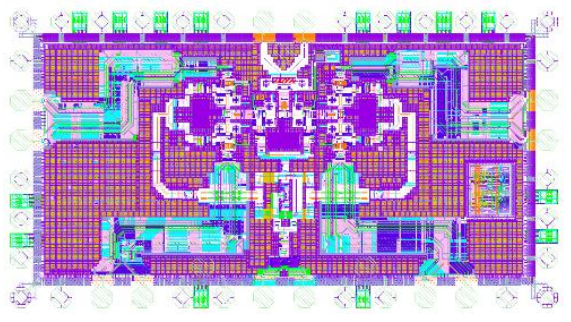

Figure 2: Layout of the SiGe BiCMOS interleaver.

This 4-to-1 interleaver is inherently capable of equalizing the signal and can be configured in three different ways, thus increasing the versatility of the component. First, 4-to- 1 interleaver operation can be used to time-interleave four 32 Gbaud analog signals into one 128 Gbaud signal allowing direct generation of 128 Gbaud Pulse Amplitude Modulation (PAM4) signals from four 32 Gbaud PAM4 signals, or 2-1 interleaver operation can be used to time interleave two $64 \mathrm{~Gb} / \mathrm{s}$ analog signals into a high speed 128 Gbaud data stream. This implementation of the interleaver can be configured to scale and add the input signals, instead of performing the interleaving action. As such, it can also be used to generate $128 \mathrm{Gbaud}$ PAM4 signal from four 64 Gbaud Non-Return-to-Zero (NRZ) signals, allowing different possibilities for the generation of the electrical data.

Regarding the architecture of the SiGe BiCMOS DAC simulation experiments indicate that a non-uniform distribution of the DAC levels can increase the signal quality. Furthermore the DAC can be configured to receive either six $64 \mathrm{~Gb} / \mathrm{s} \mathrm{NRZ}$ streams or six 32 Gbaud PAM4. The latter will ensure compatibility with emerging standards such as CEI-56G-PAM4 and IEEE P802.3bs, which will also be supported by next generations field programmable gate arrays (FPGAs).

\section{InP-HBT electronics}

Linear drivers (DRV) in InP with frequency peaking have been designed. In Figure 3, layout and simulations of a representative DRV test circuit are presented, showing broadband $(>100 \mathrm{GHz})$ frequency response with positive slope up to $80 \mathrm{GHz}$ intended to compensate frequency roll-off from the expected environment. Simulated PAM-4 eye diagrams at 
70 GBd show suitable linearity of the test DRV along with effective eye opening of an input signal filtered by a $5^{\text {th }}$ order Bessel filter with $-3 \mathrm{~dB}$ attenuation at $20 \mathrm{GHz}$.

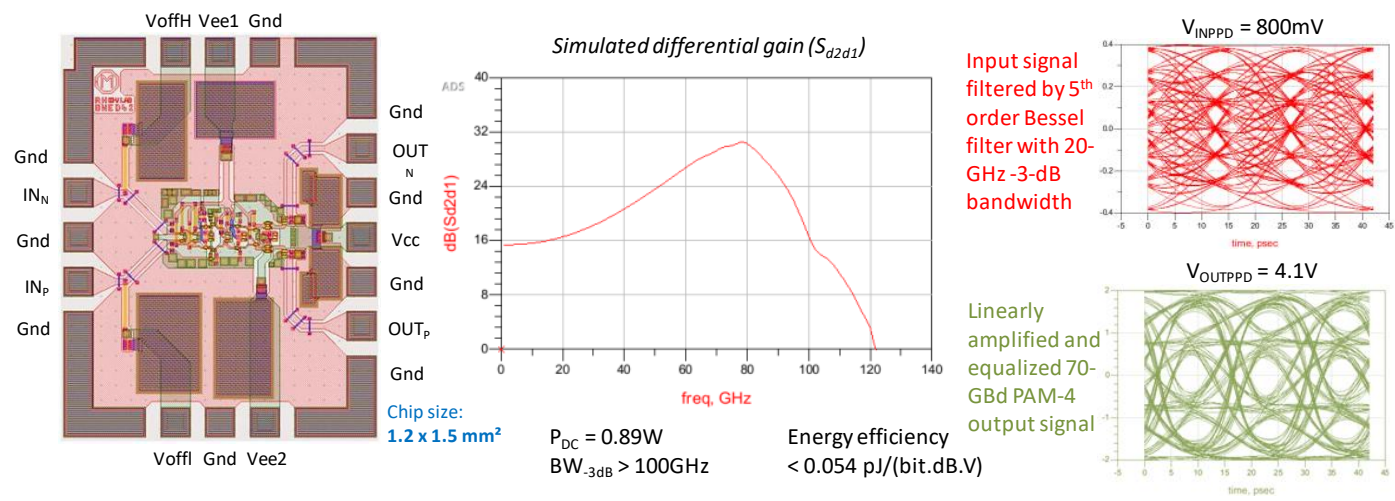

Figure 3: Layout of InP-HBT electronics IC Linear driver test circuit, simulated $\mathrm{S}_{\mathrm{d} 2 \mathrm{~d} 1}$ and PAM-4 eye diagrams.

Analog signal interleaver (A-MUX) circuits on the InP-HBT platform are also developed within QAMeleon in order to allow compatibility with both CFP2-DCO and CFP2-ACO form factors. In the first case, SiGe BiCMOS DACs and interleaver integrated circuits (ICs) are co-developed on the same chip and used together with InP-HBT linear drivers and in the second case, InP-HBT interleaver and driver are co-developed on the same chip and used with external SiGe BiCMOS DACs. At the receiver, InP-HBT de-interleaver and transimpedance amplifier (TIA) circuits are deployed.

\section{InP photonic components for data generation and reception}

QAMeleon has selected the high performance InP Mach Zehnder Modulator (MZM) technology platform for the development of high bandwidth IQ MZM structures in order to generate the 128 Gbaud optical data flows. A new NPIN and NIPN layer stack in InP with optimized metal/semiconductor interfaces that would support the 3-dB electro-optic bandwidth requirements of higher than $70 \mathrm{GHz}$ and drive voltage requirements below $2 \mathrm{~V}_{\mathrm{pp}}$ is developed. A capacitively loaded travelling wave electrode (TWE) configuration with a dual-drive electrode design will be employed in order to minimize radio frequency (RF) loss beyond $50 \mathrm{GHz}$, with low insertion losses as well as to minimize RF crosstalk between the I and Q tributaries of the RF pulse steams that drive the modulator. A NPIN deep ridge MZM structure has been evaluated through simulations versus the standard PIN structure and the results are presented in Figure 4 below.

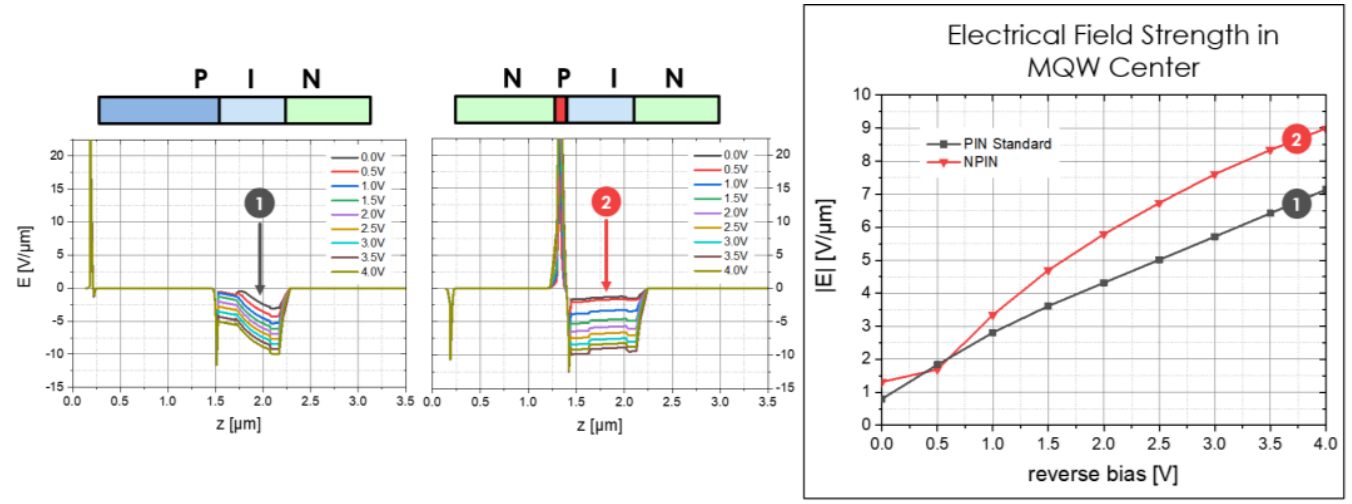

Figure 4: Electrical Field Strength in the MQW Center vs reverse biasing in Volts for the standard PIN and the NPIN layer configuration targeted for QAMeleon's modulators.

The NPIN epitaxial (EPI) structure (Figure 5 (LEFT)) will be driven based on a differential driving scheme with impedance of the TWE at 2 × 25 Ohms and has stringent space requirements at the RF interface. As shown in Figure 5 (RIGHT), the $3-\mathrm{dB}$ bandwidth of the new NPIN modulator structure is expected to be more than $70 \mathrm{GHz}$. QAMeleon is also investigating a new NIPN structure in tandem which is expected to yield lower swing voltage and electro-optical insertion loss. QAMeleon transmitter will integrate polarization handling elements on the InP photonics platform yielding compact dual polarization-enabled devices. 

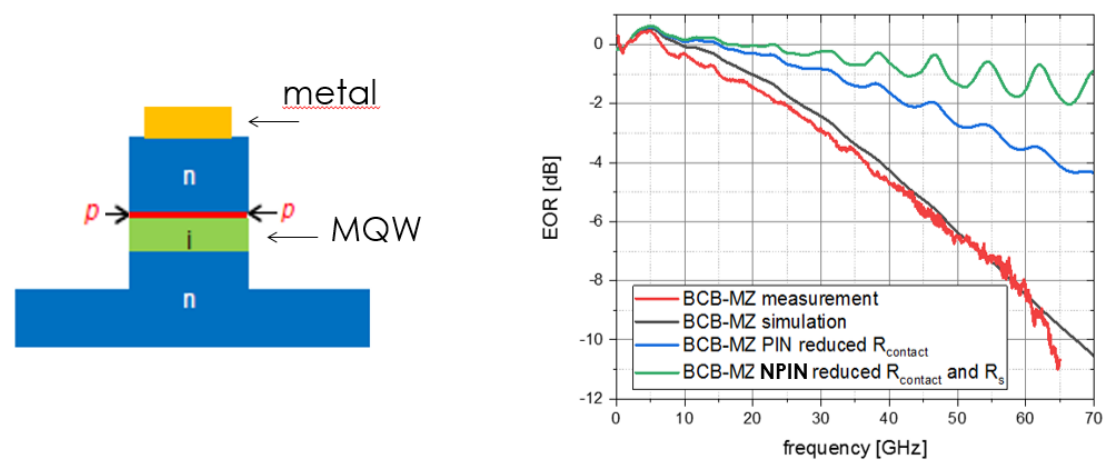

Figure 5: (LEFT) InP NPIN Deep Ridge waveguide structure; (RIGHT) RF simulation of a new designed TWE on BCB together with the new NPIN EPI-waveguide design compared to standard MZM measurement

The coherent receiver will follow current industry standards and will employ dual 90 degrees hybrids, monolithically integrated high bandwidth Photodiodes (PDs) with more than $110 \mathrm{GHz} 3-\mathrm{dB}$ bandwidth. The responsivity is expected to be $0.08 \mathrm{~A} / \mathrm{W}$ at each PD for the wavelength of $1550 \mathrm{~nm}$.

\section{Narrow Linewidth Laser}

QAMeleon will develop laser sources for coherent communications with ultra-narrow linewidth on InP that will used for feeding the InP IQ MZM and the 90-degree optical hybrids at the receiver. The laser design is based on a modulated grating distributed Bragg reflector (MG-DBR) structure in order to achieve the high-performance requirements on linewidth, output optical power and noise response. Figure 6 illustrates the cross section of the laser design. The laser will be fully tunable throughout the $\mathrm{C}$-band, its linewidth will be $100 \mathrm{GHz}$ and the output power $+19 \mathrm{dBm}$. In addition, higher operation temperature will be pursued in order to reduce the overall power consumption of the optical Tx/Rx subsystems. Furthermore, improvements on the process side will be performed in order to reduce optical losses in the cavity and improve the electrical isolation processes. Design optimization will be performed targeting high production yield and proven reliability required by commercial application products.

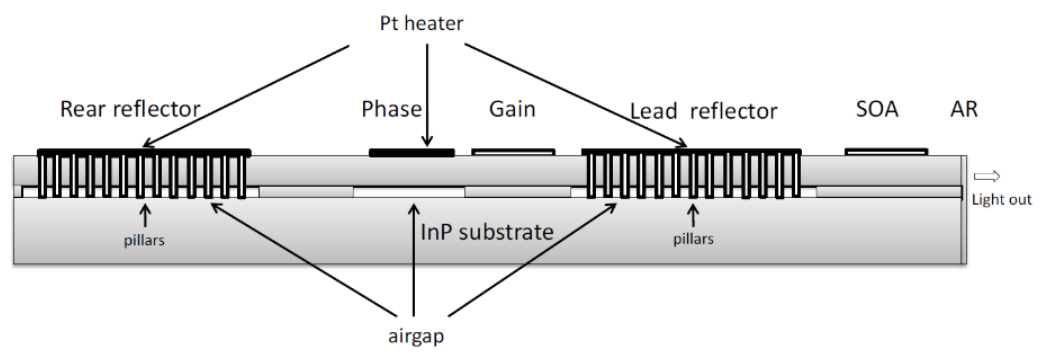

Figure 6: Cross-section of QAMeleon InP narrow linewidth laser.

The estimated overall power consumption of QAMeleon transmitter considering all its comprising electronics and photonics components is $14.7 \mathrm{~W}$ at $25^{\circ} \mathrm{C}$ including thermoelectric cooler (TEC) for the Narrow Linewidth Laser (NLL) and the IQ modulator while for the QAMeleon receiver is $11.1 \mathrm{~W}$ at $25^{\circ} \mathrm{C}$.

\section{QAMELEON ROADM CONCEPT}

QAMeleon is developing the core photonic technology to address the key challenges faced by network operators concerning the main building blocks of Colorless, Directionless, Contentionless and Gridless (CDCG) ROADMs i.e. WSSs and TPAs. Port scalability is of outright priority, because each node must cope with a large number of ports and wavelength division multiplexing (WDM) channels. This is reflected in relevant market studies, indicating large-port-count WSSs $\left(1 \times 10\right.$ or larger) as the fastest-growing area among ROADM components ${ }^{[21]}$. Scaling the number of ports can dramatically reduce the number of WSS modules in a node, avoiding cascades of switches, as shown in Figure 7 . Note that even when 
large-port-count WSSs are deployed, a substantial number of WSS modules are required per ROADM. This in turn raises the need for miniaturization, to save operator's real estate.

QAMeleon ROADM concept is based on the hybrid integration of InP photonic integrated circuits (PICs) with Liquid Crystal on Silicon (LCoS) technology using an Electro-Optic Polymer Board (EOPCB) replacing bulky free-space configurations. Two types of WSS elements are being developed within the project, i) a 1x24 hybrid InP PIC-LCoS WSS and an 8x24 TPA based on the same technology approach and ii) a fully integrated 1x4 WSS with ultrafast switching times based on the use of InP Semiconductor Optical Amplifier (SOAs) with nanosecond gain dynamics. In both cases the EOPCB acts as the host board for the intimate integration of InP PICs and the mechanical stability of free space optical components.

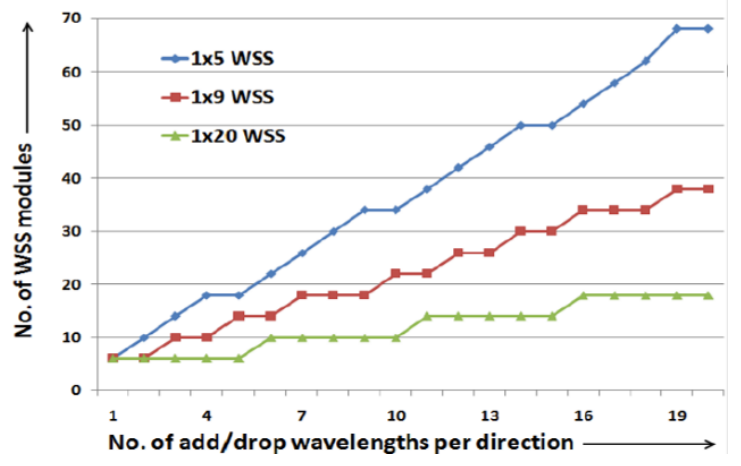

Figure 7: Number of WSS modules in a 4-degree node as a function of dropped wavelengths per direction ${ }^{[22]}$.

\section{Hybrid InP-LCoS 1x24 WSS and 8x24 TPA}

Regarding the 1x24 WSS, QAMeleon has opted for a hybrid InP-LCoS architecture which is based on InP wave frontends (WFE), a micro-optics free space setup implemented on a Polymer Electro optical (EO) motherboard and a commercial LCoS panel (Figure 8). The input signal is introduced from the fiber into the input polymer waveguide (PWG) and then directed into the respective InP waveguide and the polarization beam splitter/combiner (PBSC). At the PBSC output the light is split into two different polarization paths. The TM polarized signal must be rotated before entering the WFE since the LCoS operation is polarization sensitive. The WFE relies on an AWG with zero-path length difference in order to provide a plane wave output that is launched into the free space optics (FSO) setup comprising a diffraction grating (analyzing the wavefront into wavelengths), a focusing lens (focusing separated wavelengths onto discrete locations on the LCoS panel) and the LCoS device used for the beam steering. The wavelength separation performed by the Diffraction grating and the beam steering performed by the LCoS are realized in two directions perpendicular to each other allowing each beam projected on the LCoS to be independently switched with respect to each wavelength, so that it is coupled to the desired output WFEs thusly switching to the desired PWG output. 


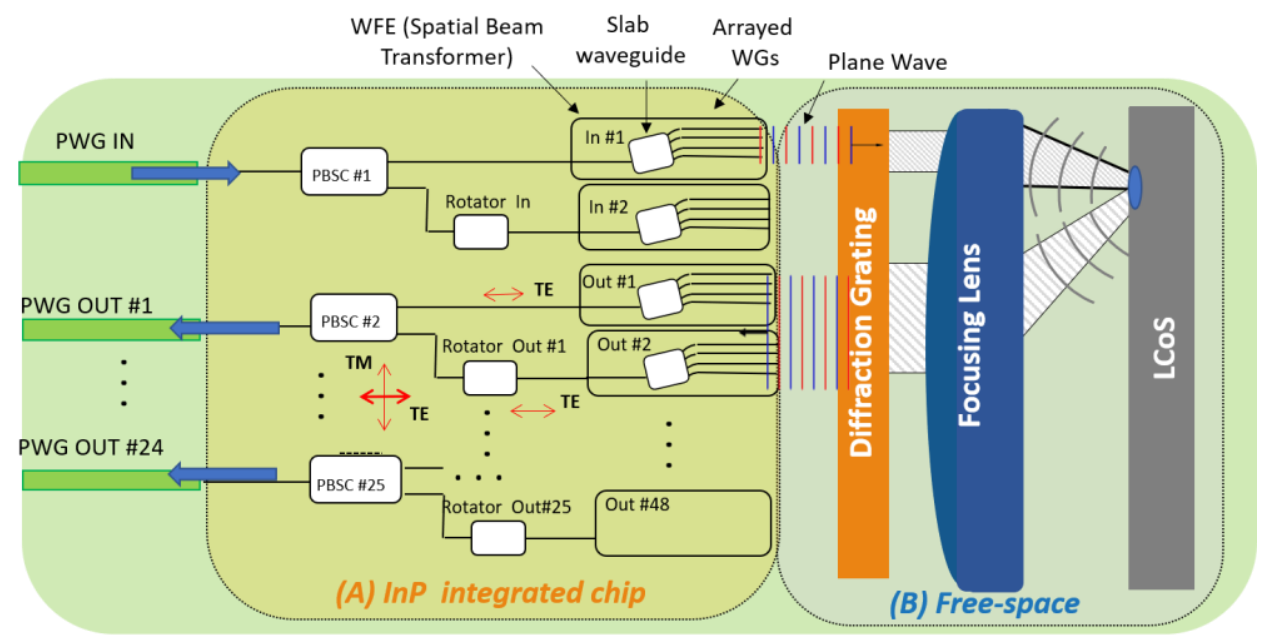

Figure 8: The QAMeleon's Hybrid $1 \times 24$ WSS architecture relying on InP WFEs along with micro-optic free space components, and a commercial LCoS Spatial Light Modulator (SLM). The motherboard for the integrated WSS device is EOPCB platform.

The key building block of these two modules is the InP WFE which replace bulky input/output (I/O) fibers. Optimization of the WFE design is based on having a beam profile that would sufficiently cover discrete areas of the LCoS panel while effectively suppressing side lobes, by altering design elements of the waveguide array: a) the number of waveguide elements, b) the spacing between the waveguides for a given wavelength operation and c) the waveguide's width. Simulation studies regarding the optimization of the WFE design are on-going. Footprint reduction is also achieved by means of the integrated polarization handling elements, PBSC and Polarization Rotators which realize the handling of both polarization states using a single LCoS panel. The PBSC design is multi-mode interference based (MMI-based) and it is shown in Figure 9. Preliminary simulations show good performances for transverse electric (TE) and transverse magnetic (TM) modes. Optimizing the MMI length and width, the shallow etch gap width as well as the taper characteristics at the input waveguide-MMI interface, concurrent maximum power of TM at Port 1 and TE at Port 2 should be achieved. The calculated Insertion Loss (IL) is less than $0.4 \mathrm{~dB}$ for both polarizations, achieving the targeted $1 \mathrm{~dB}$ value and a calculated extinction ration (ER) of the splitter of more than $20 \mathrm{~dB}$.
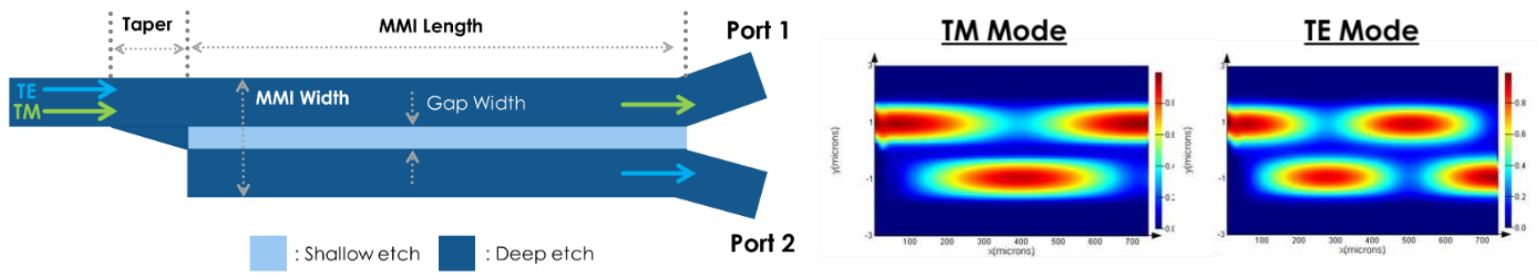

Figure 9: MMI-based PBSC component top view (Left) and Beam profile at the output of the PBSC for the TM and TE modes, respectively (Right).

The $8 \times 24$ TPA is based on the extension of the logical topology of the 1x24 hybrid WSS that combines 8 WSSs with 24 $8 \times 1$ multicast switches (MCSs) using a single LCoS. More specifically, upgrading the InP WFEs of Figure 8 with multiple input waveguides allows introduction of multiple optical signals in the WFE. A salient characteristic of this design stems from the WFE's phase characteristics: Light that is introduced from the center waveguide, exits the chip as a planar wave, perpendicular to the chip's facet. Changing the input to another waveguide tilts the output wavefront by an angle that depends on the input waveguide. As a result, after propagating through the bulk optics, the signals entering from different waveguide inputs of the WFE, are projected on different areas of the LCoS SLM. This allows combination of multiple WSSs in approximately the same footprint, maximizing the gain in integration density brought by the WFEs. Figure 10 illustrates the concept of InP WFE with multiple I/O waveguides allowing superposition of multiple WSS elements on the same LCoS panel. 


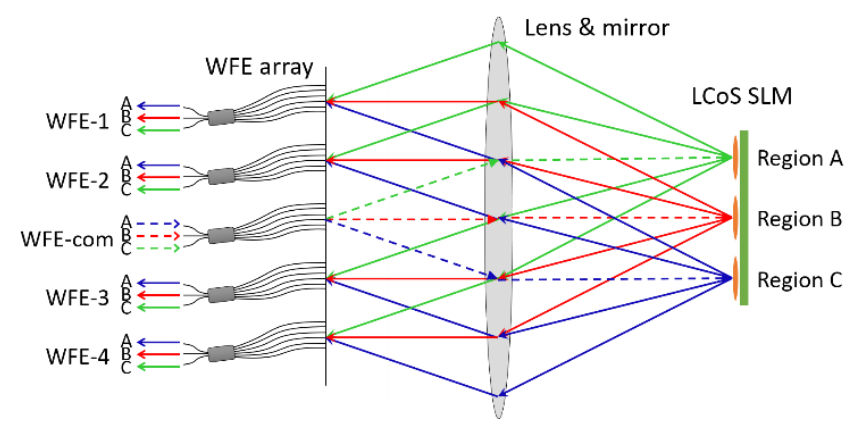

Figure 10: Multi-I/O WFE interfaces for QAMeleon 8x24 TPA.

\section{Fast 1x4 WSS}

QAMeleon 1 4 fast WSS module is based on the hybrid integration of InP PICs on the EOPCB platform allowing for a fully integrated device. At the input of the $1 \times 4 \mathrm{WSS}$, a signal is broadcasted by a 1:4 coupler. Its fundamental building block is the Wavelength Blocker (WBL) InP PIC which comprises a pair of arrayed waveguide gratings (AWGs) with $12.5 \mathrm{GHz}$ spacing and SOAs acting as ON/OFF switches in between, as shown in Figure 11 (a) and (b). For scaling the number of spectral slices (channels) multiples units of WBLs are designed into a single InP PIC. The SOAs provide extra advantage of on-chip amplification thereby compensating the losses introduced by the two AWG in the WBL and coupling losses. The SOAs also enable fast switching providing a switching time less than $20 \mathrm{~ns}^{[18],[19],[20]}$. An AWG multiplexer is used to combine outputs of an InP PIC into the output of the 1x4 WSS as shown in Figure 11 (a)).

Figure 11 (c) illustrates the "split and combine" concept of the WSS based on which selective number of AWG outputs are set to the ON and OFF state depending on the bandwidth of the incoming input signal. The incorporation of multiple AWG channels allows control of the passband width of the output WSS port with granularity of $12.5 \mathrm{GHz}$ slices.

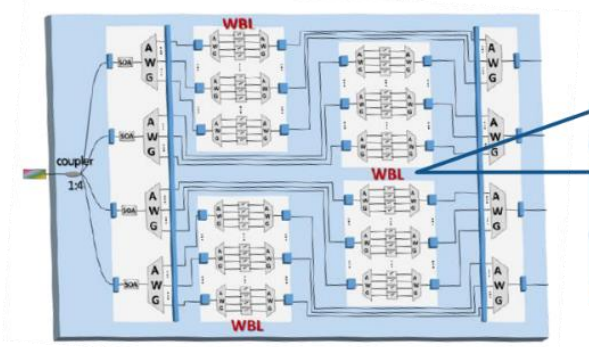

(a)

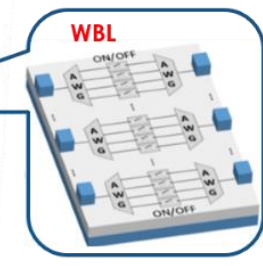

(b)

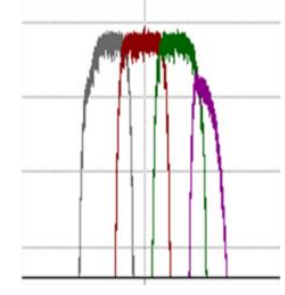

32 Gbaud

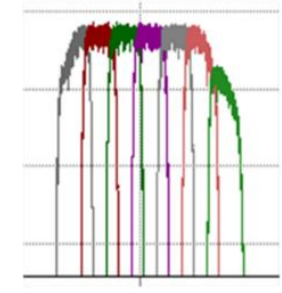

64 Gbaud

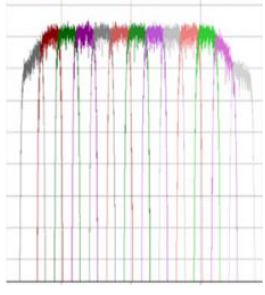

128 Gbaud

(c)

Figure 11: (a) Architecture of QAMeleon fast 1x4 WSS, (b) block diagram of WBL and (c) Bandwidth discretization of 32, 64 and 128 Gbaud in $12.5 \mathrm{GHz}$ spectral slices.

Moreover, the AWGs and the SOAs on the InP platform are targeted to be polarization insensitive devices and different waveguide geometries are under investigation based on the birefringence they induce on both TE and TM polarizations. Recent simulation studies for the birefringence of deep and shallow waveguides as function of their width, at the target wavelength of $1555 \mathrm{~nm}$, imply that polarization insensitivity of the AWGs could be obtained by controlling the width of the deep waveguides. Mechanical strain can influence birefringence too indicating that achieving the polarization insensitive condition is very susceptible to waveguide width variations. hence it is necessary to use a high-resolution lithography tool. These properties result in very sharp and uniform waveguides as can be seen in Figure 12 where "W" indicates a $300 \mathrm{~nm}$ separation between the AWG waveguides, which largely determines the channel separation. The better waveguide definition also has a positive effect on propagation losses. 


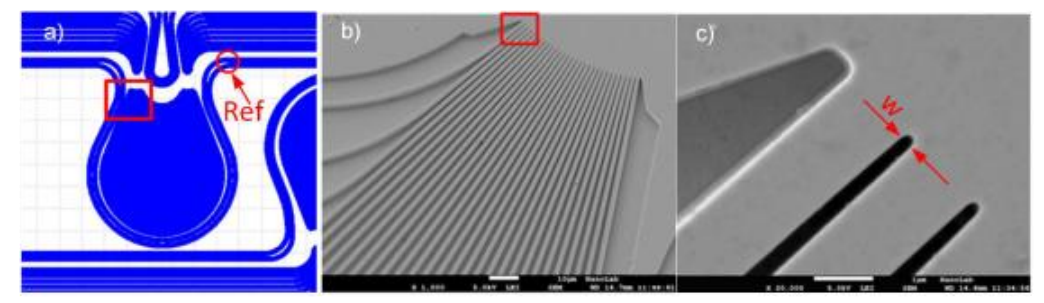

Figure 12: AWG fabricated using a high-resolution lithography tool.

\section{EOPCB platform}

The EOPCB technology offers low-loss waveguides and tight bends and acts as a host platform for the InP PICs and the free-space micro-optics setup including the LCoS. Having in mind the large number of optical components that comprise QAMeleon WSS elements, a modular approach will be followed allowing to address integration challenges and ensure scalability towards a larger number of I/O ports at reduced complexity and with moderate increase in footprint.

Optimization of the EOPCB platform relies on the current polymer material and the dimensions of the waveguides. The mode field diameter (MFD at 1/e2) is a critical parameter that affects coupling losses to the PICs (approx. $4 \mu \mathrm{m}$ MFD) and the glass fiber (approx. $10.4 \mu \mathrm{m}$ MFD) and its lower limit is given by the refractive index contrast of the polymer waveguide. The waveguide is represented by a channel type configuration with a rectangular, step-index cross section embedded in the cladding (Figure 13 Left). The MFD of the polymer waveguide depends on the physical dimension of the waveguide core and refractive index contrast of the polymer (Figure 13 Right). Currently a lower boundary of the MFD of approximately $4.7 \mu \mathrm{m}$ is anticipated and is limited mainly by the optical properties of the optical polymer. In order to minimize coupling losses further investigation in required in the potential of lateral MFD adaptation. The manufacturing of the polymer waveguides allows for an adaptation of the lateral MFD-width, which is intended to be used for reducing mode field mismatch at the fiber coupling interface. The electrical layers of the EOPCB consist of a material layer with enough conductivity and thickness for the anticipated current strength with coplanar electrical waveguides (CPW) providing the required rise and fall times.
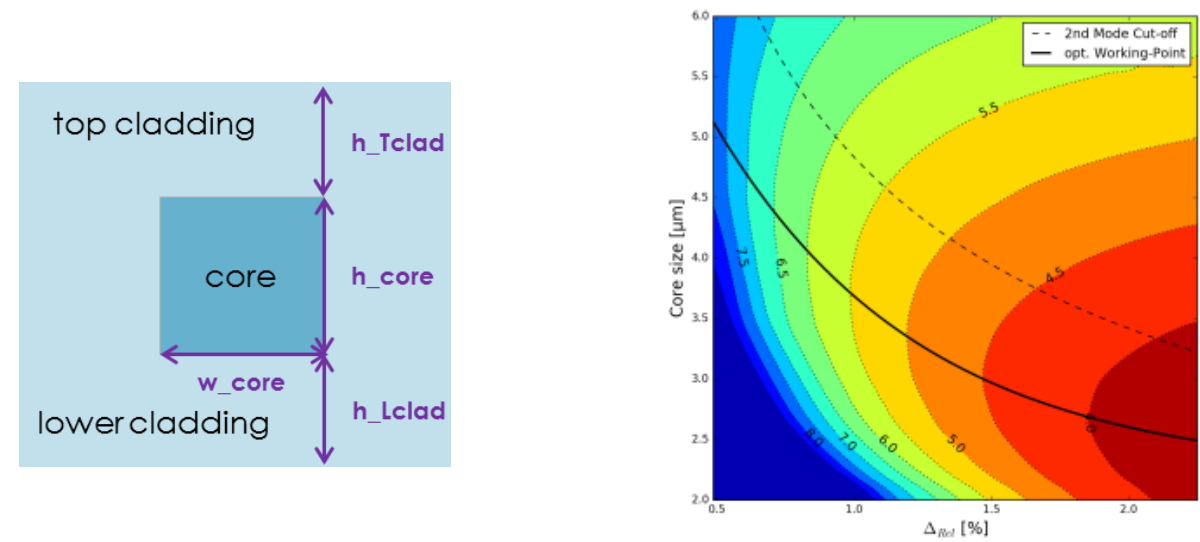

Figure 13: (LEFT|) Sectional view of channel type polymer waveguide with critical dimensions, and (RIGHT) graph of MFD as function of refractive index contrast and waveguide core size.

Within the premises of QAMeleon, various polymer waveguides with different core widths have been fabricated allowing for preliminary evaluation of the EOPCB platform with respect to MFD and optical coupling (Figure 14). The waveguide propagating loss at $1550 \mathrm{~nm}$ is $0.55 \mathrm{~dB} / \mathrm{cm}$ using the cut-back method while no excess bending radius loss is observed to radius down to $7 \mathrm{~mm}$. 
(a)

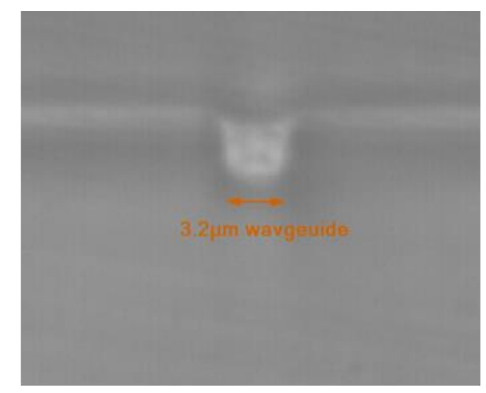

(b)

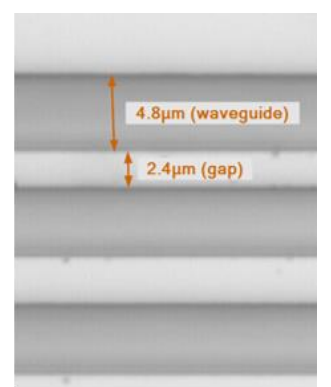

Figure 14: (a) cross-section of $3.2 \mu \mathrm{m}$ wide waveguide core, (b) top-view of waveguide cores separated by a $2.4 \mu \mathrm{m}$ wide cladding gap.

\section{Hybrid integration of InP PICs to EOPCB}

The InP-to-Polymer waveguide optical interface will be butt-coupling for simplicity and to avoid further changes in the InP platform that would be needed for an adiabatic coupling. The optimum coupled power from InP rib waveguides (cross section $2 \times 1 \mu \mathrm{m}$ ) to polymer waveguides (cross-section of $3 \times 3 \mu \mathrm{m}$ ) was evaluated through finite-difference time-domain (FDTD) simulations based on power overlap integral (OI) calculation ${ }^{[17]}$. First results yielded coupling efficiency of $25 \%$ by optimizing the InP waveguide width to $3.5 \mu \mathrm{m}$. New spot size converter are being developed in the InP platform to interface standard InP rib waveguides with single-mode fibers that can be also exploited for the interface with the polymer waveguides as envisaged within QAMeleon. Using these spot size converters preliminary results indicate that coupling efficiency can be improved up to $59.2 \%$ (2.277 dB coupling losses) and 65.97\% (1.8 dB coupling losses) for TE and TM modes, respectively without sacrificing the single-mode operation (Figure 15).
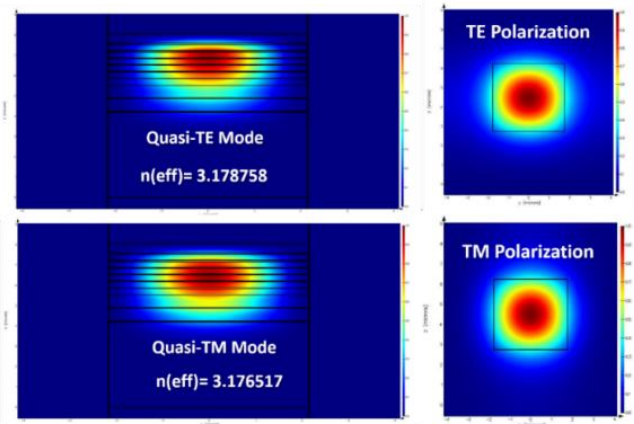

Figure 15: Showing TE and TM mode profiles using the new InP spot size converter and $3.5 \times 3.5 \mu$ m polymer waveguide.

Finally, regarding the integration and assembly process for QAMeleon WSSs and TPA modules, a flip-chip approach is considered. The target mechanical accuracy of the flip-chip process is $1 \mu \mathrm{m}$ across the horizontal and the vertical planes which imposes challenges for the vertical alignment of InP waveguide with the polymer waveguide. QAMeleon approach is based on a vertical offset on the EOPCB to control the height of the PIC (in vertical-plane) assuming the PIC is positioned 'face-down', as shown in Figure 16 (a). The tolerance of the PIC epi-layers can typically be controlled to 100's nm range. Electrical connectivity from the PIC to the EOPCB by wire bonding is preferred. The model below suggests the use of a ceramic interposer that would electrically connect to the InP via bumpers and via wire bonds to the EOPCB as shown in Figure 16 (b). The ceramic interposer will also provide mechanical support to the InP PIC.

(a)

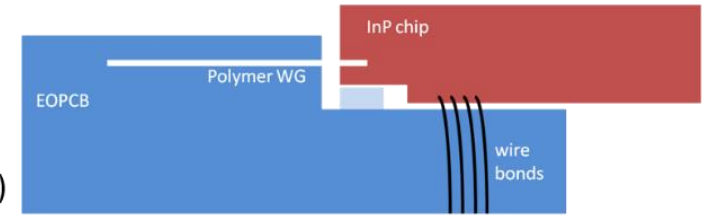

(b)

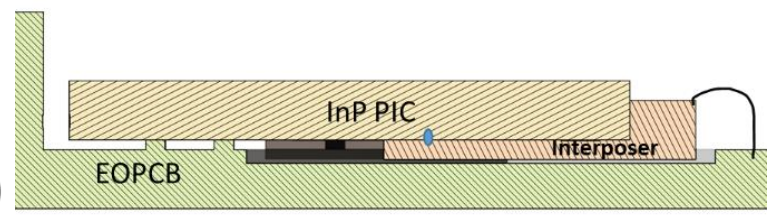

Figure 16: (a) Flip-chip assembly of InP PICs on the EOPCB of VARIO that will act as the motherboard for QAMeleon WSSs and TPA modules, (b) cross section showing the use of a ceramic interposer to facilitate electrical connectivity between the InP PIC and EOPCB and provide structural support to InP. 


\section{QAMELEON SDN AND WHITE-BOX DEMONSTRATORS}

QAMeleon control plane architecture is based on a centralized SDN controller that implements core functions related to the monitoring and programming of the underlying optical network, like the management of its topology, the maintenance of its status and the collection of its statistics, as well as the configuration of its devices through a flow-based paradigm, as shown in Figure 17 (a). The logic of the network programming is placed in dedicated SDN applications running on top of the controller. The interaction between the controller and the applications is based on REST Application Programming Interfaces (APIs) that expose the underlying optical resources with a suitable level of granularity and abstraction. The specific characteristics of the different optical devices are handled through dedicated SDN plugins, placed at the southbound of the controller and in charge of managing the interaction with the data plane. The SDN plugins implement the device-specific information models and interact with the single network elements placed at the data plane through the mediation of SDN agents, which act as translators of control plane protocol messages and configure the single elements, e.g. programming an FPGA (Figure 17 (b)).

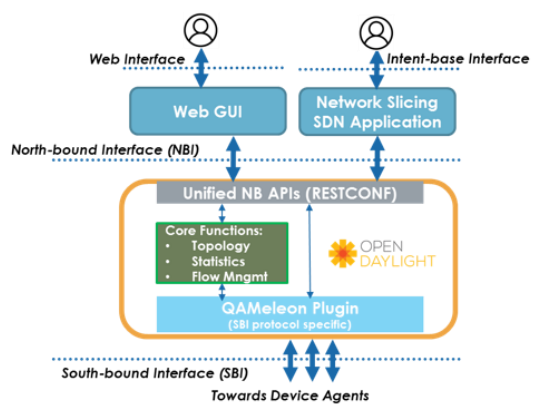

(a)

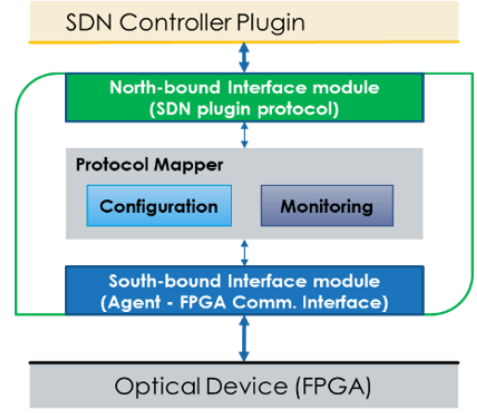

(b)

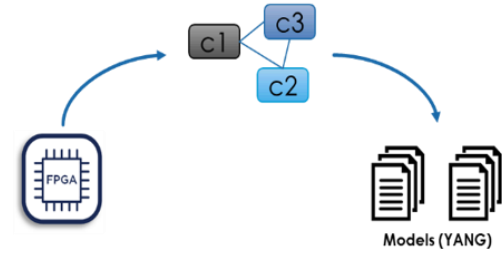

(c)

Figure 17: (a) QAMeleon functional control plane architecture, (b) SDN-Agent building blocks and interfaces, (c) Optical device represented as an FPGA: components c1, c2 and c3 of the hardware are individually modelled (example of disaggregated approach).

Following the latest trends in SDN architectures for optical networks, the QAMeleon control plane architecture strongly relies on the "disaggregation" paradigm, defining different level of abstraction and/or aggregation to describe, monitor and program single optical components, devices or network topologies as a whole. The concept of "disaggregation" is largely used in the context of the optical networks in order to simplify and automate network operations and guarantee multivendor interoperability. QAMeleon's approach is to consider an optical device as a proper aggregation of optical functions or subcomponents that are individually modelled and individually controllable. The decomposition of an optical device in a set of standard modelled subcomponents leads to the development of fully disaggregated white boxes, where software and hardware are separated (sw/hw disaggregation) and may be composed of devices from different vendors and optionally managed by different open source software.

In Figure 17 (c), a device (generically represented as an FPGA in the picture) is decomposed in a set of components, which are modelled using YANG language. The interaction between the single components, properly modelled, represents the behavior of the device as a whole. For example, a ROADM is modelled as a set of interconnected WSSs and TPAs, where each of them is controllable and/or configurable as single functional entities.

The communication interface of the SDN agent and QAMeleon transponder and ROADM devices will be vendor specific, while the interface between SDN controller and SDN agent will be based on the standard NETCONF protocol. Both the interactions will be based on a client to server communication approach. More specifically, in the controller to agent communication, the Agent will act as a server while the SDN controller, as the entity that performs data plane configuration, will constitute the client. In the agent to device communication, the agent will act as client while the device constitutes the server. The parameters that will be controlled at the transponder are the symbol rate of the optical flows, the modulation format and the wavelength per generated optical flow yielding a fully flexible S-BVT transponder. As for the WSS and TPA modules, the reconfigurable SDN parameters will be the I/O port, the center frequency and the passband 
width. The above parameters will be translated in device-specific parameters that will configure the operation of the optical hardware. Figure 18 illustrate the S-BVT and ROADM "white-box" demonstrators.
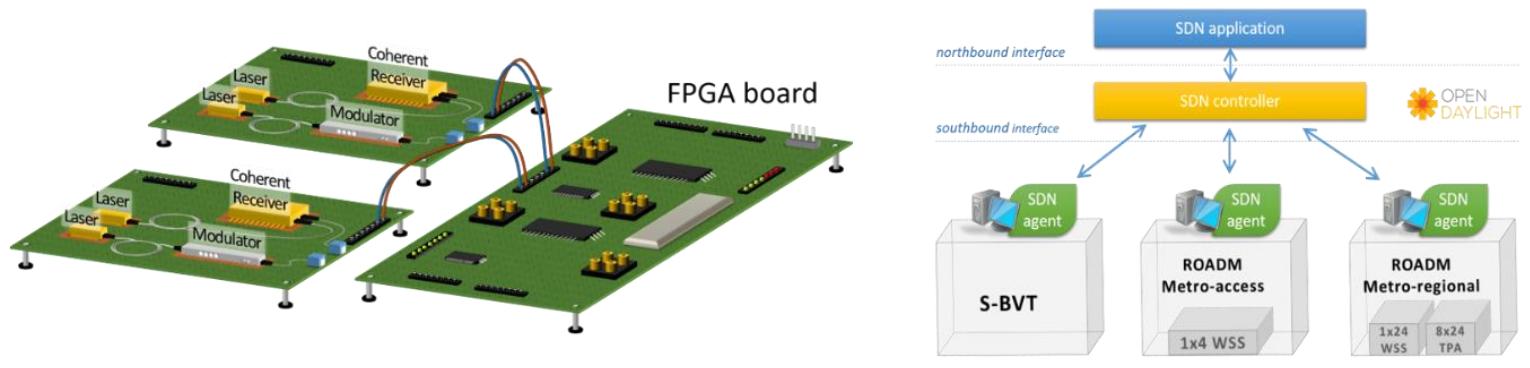

Figure 18: QAMeleon S-BVT and ROADM white-box demonstrators.

\section{MODULATION FORMATS \& DSP}

In order to achieve QAMeleon's objectives of next-generation elastic optical networks (EONs) operating in the flexible Dense WDM (DWDM) grid $^{[6]}$, programmable, rate-adaptive operation is necessary. The QAMeleon transceiver should support different modulation formats with different spectral efficiencies, adapting the bit rate and reach based on actual network traffic demand ${ }^{[7]}$. Coded modulation techniques are a viable method to enhance the total coding gain and close the gap to the channel capacity. In addition to conventional Dual Polarization (DP) M-ary Quadrature Amplitude Modulation (M-QAM) formats, QAMeleon has devoted significant effort in investigating the application of fourdimensional (4-D) modulation, probabilistically shaped (PS) M-QAM, as well as digital subcarrier multiplexing to provide the required flexibility for the S-BVT.

4-D modulation in coherent optical transmission systems uses both the real (in-phase) and imaginary (quadrature) tributaries in 2 orthogonal polarizations. This allows construction of power-efficient signal constellations by the optimal placement of the signal points in 4-D space, and as a result, Optical Signal to Noise Ratio (OSNR) performance can be increased. For communication systems, sensitivity gains can be attained by optimizing the signal constellation ${ }^{[8],[9]}$. In QAMeleon a particular set of 4-D formats is considered, that are constructed by applying the set-partitioning technique on DP-M-QAM formats, referred to as M-ary set-partitioned QAM (M-SP-QAM). An important aspect of M-SP-QAM formats is that they can be implemented using the same hardware as conventional DP-M-QAM formats, with some minor modifications in the digital signal processing (DSP) modulation/demodulation algorithms. Accordingly, a transceiver which supports standard DP-M-QAM in addition to M-SP-QAM, provides a wide range of possible spectral efficiencies. Thus, it enables a very good trade-off between bit rate and transmission reach. Monte Carlo simulations are performed in VPItransmissionMaker Optical Systems to estimate the required optical signal-to-noise ratio (ROSNR) for M-SP-QAM and their corresponding DP-M-QAM formats at a hard-decision (HD) forward error correction (FEC) limit of Bit Error Rate $(\mathrm{BER})=3.8 \times 10^{-3}$. The spectral efficiencies (SEs) of these formats versus the ROSNR are plotted in Figure 19. All modulation formats are operating at a fixed symbol rate of 28 Gbaud. It should be noted that for DP-M-QAM formats, it is only possible to increase the $\mathrm{SE}$ by a step of $1 \mathrm{bits} / \mathrm{symbol} /$ polarization, where each step requires $\sim 2.5-4 \mathrm{~dB}$ additional OSNR. Finer granularity of $0.5 \mathrm{bits} / \mathrm{symbol} /$ polarization in SE can be provided if the M-SP-QAM formats are also considered which serves as an intermediate step between DP M-QAM formats. This enables a smooth transition in information bit rate (each increment requires $\sim 1-2 \mathrm{~dB}$ extra OSNR) achieving greater flexibility. 


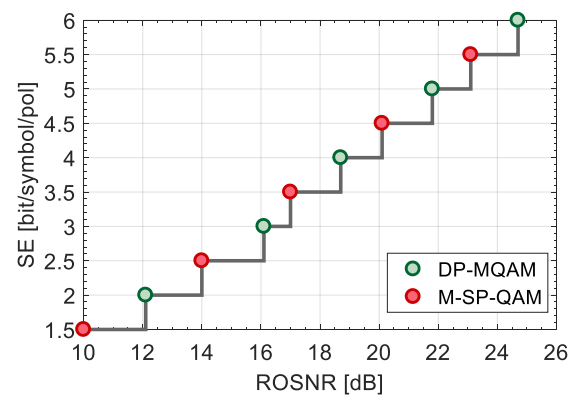

Figure 19: Spectral efficiency vs ROSNR for M-SP-QAM and DP-M-QAM formats at BER=3.8×10-3.

Probabilistic shaping (PS) is a technique used to impose a non-uniform distribution on the transmitted symbols, such that the $d_{\min }$ between constellation points is increased for the same average signal energy. This technique is used to achieve the so-called shaping gain, which is capped to $1.53 \mathrm{~dB}$ for the additive white gaussian noise (AWGN) channel ${ }^{[10]}$. PS is very attractive for optical communication systems because of the performance gains and the ability to adjust spectral efficiency (and thus, the data rate) with arbitrarily fine granularity. QAMeleon has developed DSP that generates PS square M-QAM formats. Such formats do not impose additional requirements on the hardware (e.g. in terms of DAC resolution needed), compared to conventional DP-M-QAM transmitters.

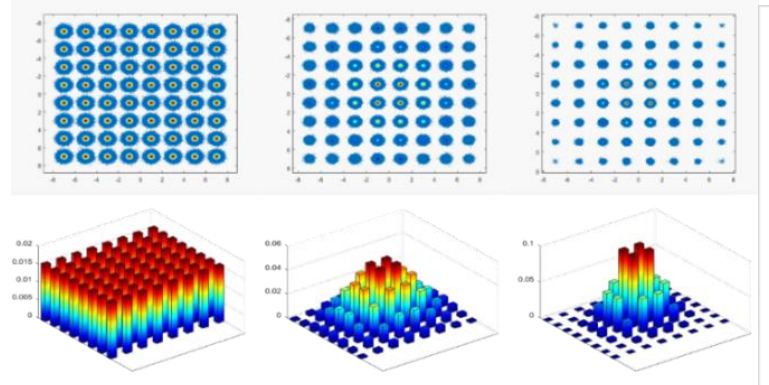

(a) $\mathrm{SE}=6.0 \mathrm{bits} / \mathrm{sym}$

(b) $\mathrm{SE}=5.5 \mathrm{bits} / \mathrm{sym}$

(c) $\mathrm{SE}=4.5 \mathrm{bits} / \mathrm{sym}$

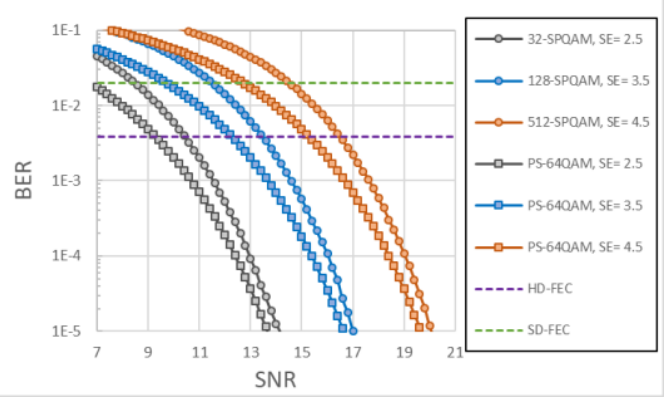

Figure 20: (LEFT) Shaped constellation diagrams for 64QAM with the corresponding PMFs for three different SEs: (a) 6.0 bits/symbol (i.e. unshaped); (b) 5.5 bits/symbol; (c) 4.5 bits/symbol. (RIGHT) BER as a function of SNR for selected M-SPQAM and PS-64QAM formats, assuming an AWGN channel.

Figure 20 (LEFT) shows the constellation diagrams for PS-64QAM with the corresponding probability mass functions (PMFs) for three different cases. As can be seen PS-M-QAM allows using a fixed base constellation, symbol rate and FEC overhead, with the SE able to be tuned simply by changing the input distribution of the signal points ${ }^{[11]}$. In a flexible optical network environment, the SE can therefore be traded off for OSNR performance (and thus, transmission reach), with very fine granularity, allowing maximization of overall network capacity. Performance comparisons through simulation of MSP-QAM formats with PS-64QAM in back-to-back configuration with AWGN as the only impairment are out. Figure 20 (RIGHT) shows the BER performance as a function of SNR for M-SP-QAM with M =32, 128 and 512, corresponding to $\mathrm{SEs}=2.5,3.5$ and 4.5 bits/symbol respectively. In addition, the performance of PS-64QAM formats at the same SEs are also plotted. PS-64QAM offers $\sim 1.8 \mathrm{~dB}$ gain over M-SP-QAM at the SD-FEC limit of BER $=2 \times 10^{-2}$, for the same SE. The gain reduces to $\sim 1.2 \mathrm{~dB}$ at the HD-FEC limit of BER $=3.8 \times 10^{-3}$. This gain gets smaller as the SNR increases, until eventually it vanishes at asymptotically high SNR. The larger gain at the low SNR regime can be attributed to the fact that M-SP-QAM employs anti-Gray mapping, and a wrongly decided symbol will not necessarily lead to only a single bit error, but in multiple bit errors. Moreover, the number of nearest neighbors for 4-D formats is larger than that of the 2-D PS64QAM format. This factor limits the achievable gain for 4-D, especially at low-SNR, and the benefit of the PS signal becomes more apparent in this region. However, this comes at the expense of higher implementation complexity. PS requires using a distribution matcher at the transmitter side and arithmetic decoder at the receiver side ${ }^{[11]}$, which increases the computational complexity of the system versus M-SP-QAM, which can be implemented using a few logical gates ${ }^{[12]}$ and a simplified symbol decision function ${ }^{[13]}$.

The framework needed to add FEC to PS-M-QAM formats is also investigated. This is important for QAMeleon to be able to approach the channel capacity of a given transmission link. Simulation of the transmitter-side DSP in order to allow 
adding low-density parity-check (LDPC) codes to the PS-M-QAM generator are implemented (Figure 21) ${ }^{[14]}$. The implementation is specifically formulated for square M-QAM formats as square QAM is widely adopted and much simpler to generate than other, arbitrary constellations.

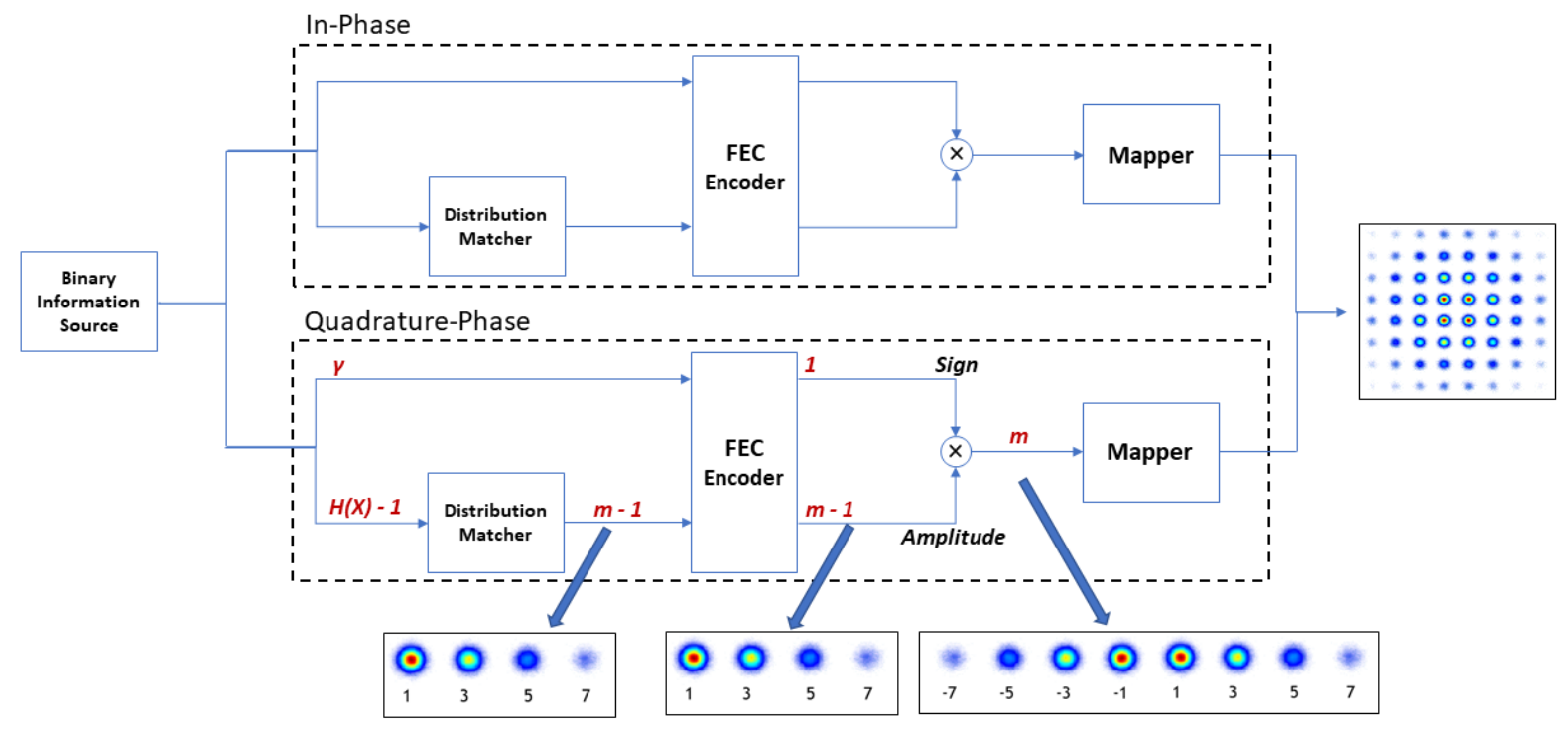

Figure 21: Architecture incorporating FEC with PS square QAM. The red text indicates the bits/symbol carried at each stage.

The distribution matcher (DM) first generates a Maxwell-Boltzmann distribution of entropy $H(X)-1$ for the positive amplitudes of an 8-ary Pulse Amplitude Modulation (PAM-8) signal (i.e., half the levels of a PAM-8). Note that the number of levels generated at this point is defined by the target base distribution to be shaped; in this example it is 64-QAM, and thus 8 levels per quadrature tributary are needed. This 'half' PAM- 8 then carries $m-1$ bits/symbol (where $m=\log _{2} 8$ ). The other (negative) amplitude values of the PAM-8 are generated from a sign bit, which carries the FEC overhead. The FEC overhead can be less than a whole bit, in which case a fraction $1-\gamma$ of the sign bit can carry information (note that $\gamma \leq 1$ ). The code rate is then given by $R_{c}=(m-(1-\gamma)) / m$, and can therefore not be less than $(m-1) / m$. The total information rate can then be written as:

$$
I R=\beta+\gamma=H(x)-\left(1-R_{c}\right) m
$$

Demodulation of DP (dual-polarization) PS-M-QAM signals poses unique challenges for the DSP of the digital coherent receiver. Algorithms that have been developed for blind demodulation of DP M-QAM formats, where uniform symbol probabilities are assumed, fail to perform adequately when applied to their PS counterparts. The problem is further compounded by the fact that PS formats are necessarily reliant on using base constellations which are high-order $(M=64$ or 256), and these are inherently more difficult to demodulate, even in the uniform case. Research into DSP algorithms for blind demodulation of PS-M-QAM, focusing first on polarization-demultiplexing and equalization, has been started. A novel approach has been developed that relies on a 2-stage scheme comprising a polarization alignment algorithm, followed by a probability-aware time-domain Multi-Input Multi-Output (MIMO) equalizer ${ }^{[16]}$. An illustrative example simulation setup that demonstrates the operation of the DSP demodulation process is presented in Figure 22. The setup consists of a dual-polarization transmitter that generates the DP PS-M-QAM signal, which is transmitted over 20 spans of standard single-mode fiber (SSMF), with lumped amplification. After coherent detection, the signal is digitized and processed with the DSP algorithms that include the new novel polarization demultiplexing and equalization scheme. The simulation includes the impairments expected in a real transmission system, including random birefringence and polarization mode dispersion (PMD), amplified spontaneous emission (ASE) noise, limited ADC resolution as well as severe bandwidth limitations to stress-test the DSP and prove its ability to cope with severe constellation distortions. Figure 23 shows constellations obtained after transmission of a DP PS-64-QAM carrying 4 bits/symbol over $1600 \mathrm{kms}$. The received OSNR is measured at $\sim 18 \mathrm{~dB}$, while the symbol error rate (SER) is estimated at $\sim 6 \cdot 10^{-4}$. 


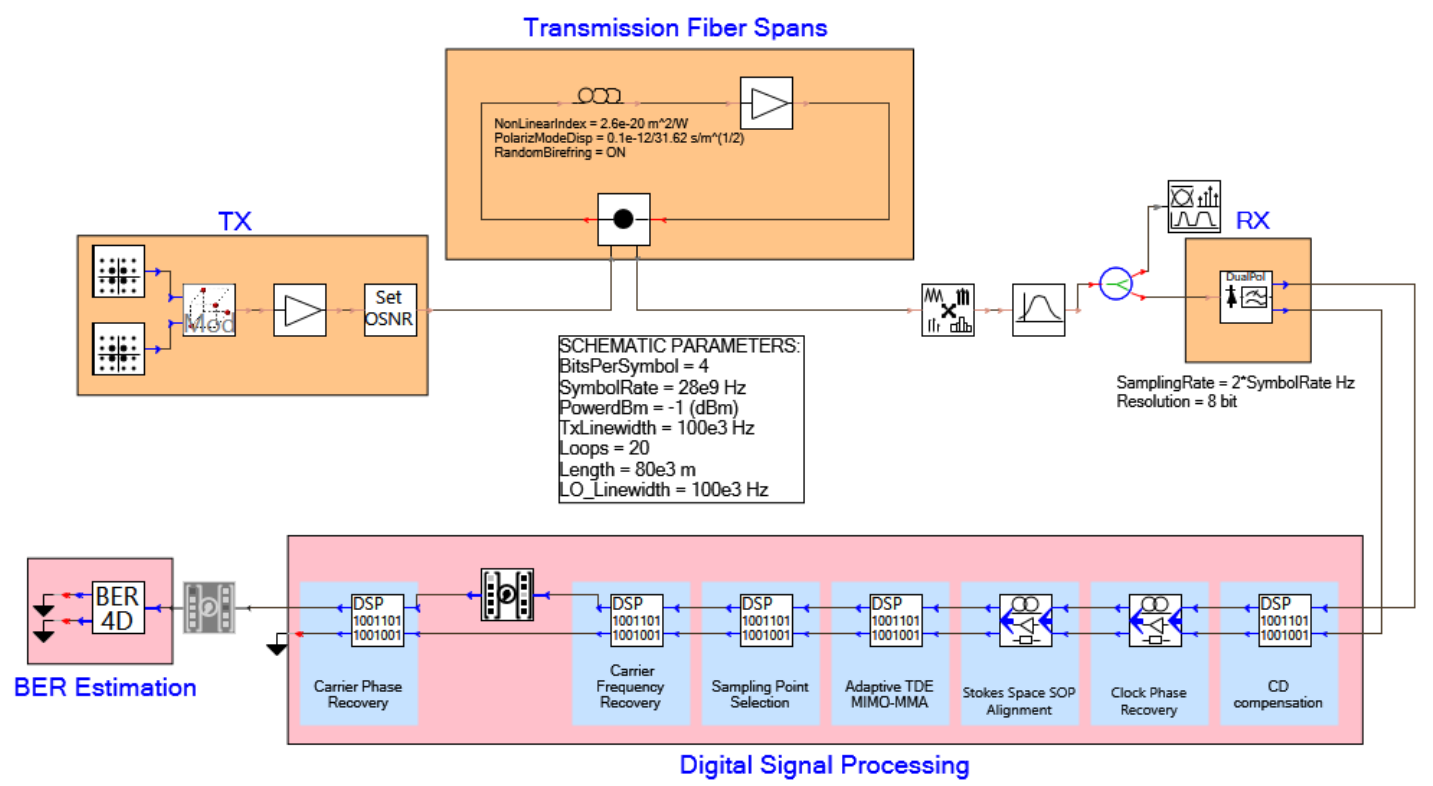

Figure 22: Simulation setup created in VPItransmissionMaker Optical Systems for the transmission, reception and demodulation of DP PS-M-QAM signals.
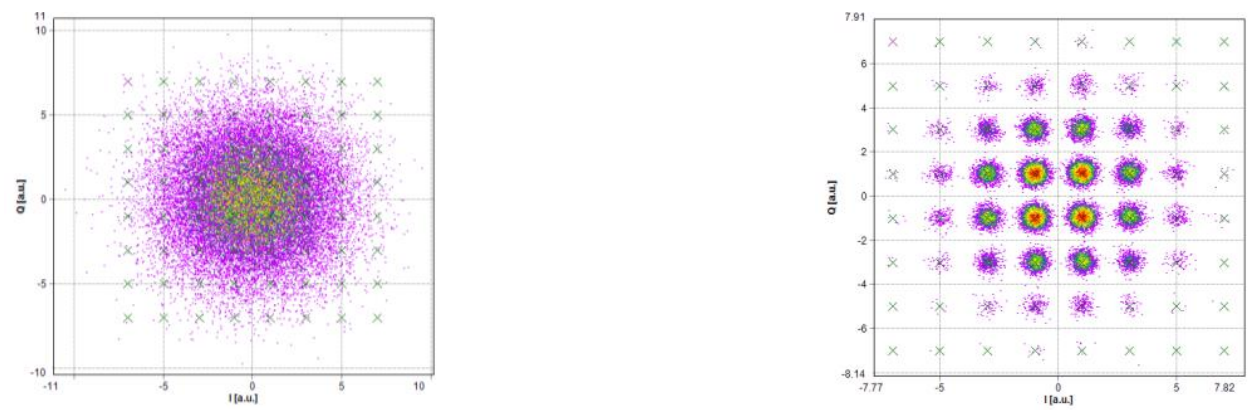

Figure 23: (LEFT) X-polarization constellation after SOP alignment, and prior to equalization. (RIGHT) The same constellation after equalization with the novel probability-aware TDE MIMO.

Regarding the Digital Subcarrier Multiplexing (SCM) scheme, preliminary studies on using standard M-QAM schemes (quadrature phase shift key (QPSK) and 16-QAM) have been performed. An Arbitrary Waveform Generator was employed to evaluate the performance of the SCM IQ streams prior to feeding them to the optical modulator for three different scenarios (Table 1).

Table 1: Initial scenarios for experimental evaluation of Digital SCM.

\begin{tabular}{|l|l|l|c|}
\hline $\begin{array}{l}\text { Proposed SCM } \\
\text { Scheme }\end{array}$ & $\begin{array}{l}\text { Format per } \\
\text { subcarrier }\end{array}$ & $\begin{array}{l}\text { Rate per } \\
\text { subcarrier }\end{array}$ & Subcarrier spacing \\
\hline $2-S C M$ & QPSK / 16-QAM & 16 Gbaud & $18.4 \mathrm{GHz}$ \\
\hline $3-S C M$ & QPSK / 16-QAM & 10 Gbaud & $9.2 \mathrm{GHz}$ \\
\hline $4-S C M$ & QPSK / 16-QAM & 8 Gbaud & \\
\hline
\end{tabular}

The appropriate roll-off filters was applied in order to get total capacity 32 Gbaud QPSK / 16-QAM in single carrier format. The generated spectrums prior to the DAC (64 GSa/s sampling rate) are presented in Figure 24. 

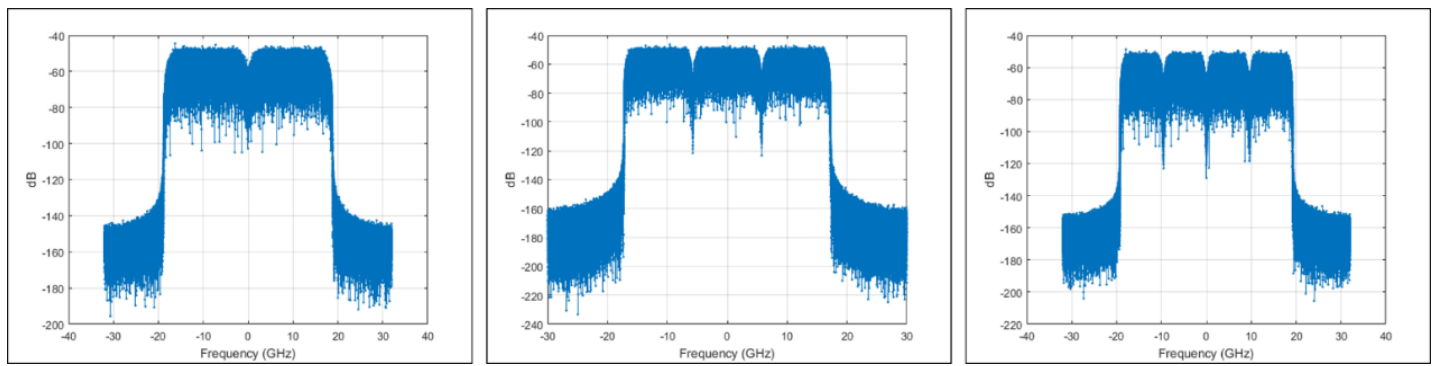

Figure 24: Digital sequence SCM spectra at the input of the DAC based on the scenarios of Table 1.

For the Tx side, bit-to-symbol mapping, pulse shaping per subcarrier and digital pre-distortion of the optoelectronic frontend using frequency domain equalizers is used. On the Rx side the DSP is shown in Figure 25. The desired subcarrier for demodulation is selected by down-converting it on the baseband and low-pass filtering is performed. The remaining DSP chain is identical to the case of demodulating a single carrier M-QAM scheme. Thus, in this first approach no shared processes for the detection of each subcarrier is used and the demodulation of each band is performed independently.

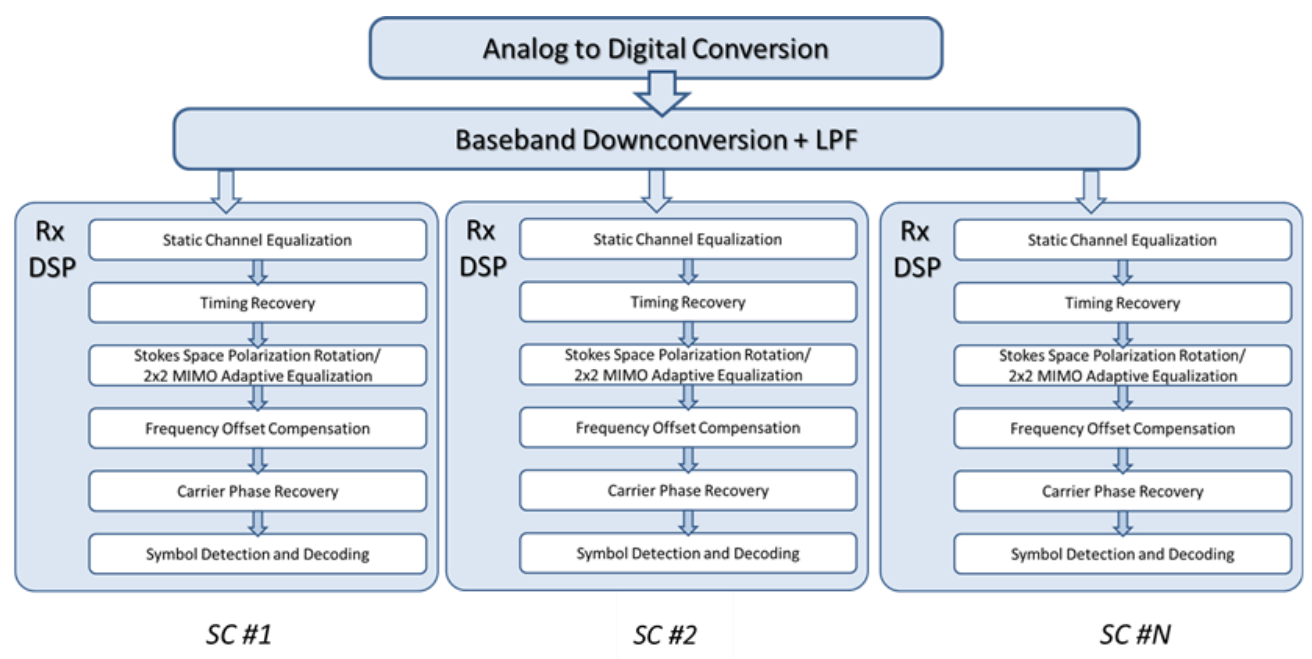

Figure 25: Rx-side DSP for SCM demodulation.

Employing a simple coherent transmitter/receiver link the combined Tx/Rx DSP for the SCM scenarios was evaluated. A single-polarization IQ stream is received by the coherent receiver, where the Digital Oscilloscope provided the digitized waveforms for Rx-side processing. The performance of the selected scenarios is depicted in Figure 26. 

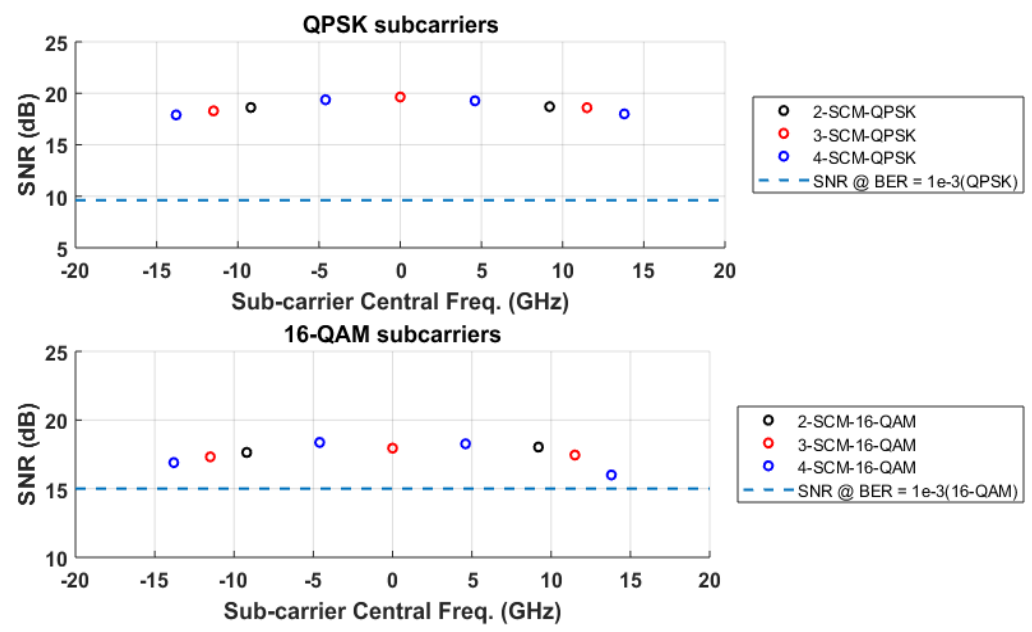

Figure 26: Performance evaluation of the selected SCM scenarios.

\section{CONCLUSIONS}

Coherent optics pose a promising solution for the ever-increasing demands in traffic which pose key challenges for network operators and vendors. The progress that QAMeleon, an EU funded R\&D project, has made towards developing a new generation of faster and greener sliceable bandwidth-variable electro-optical transceivers and WSS switches able to handle up to 128 Gbaud optical signals carrying flexible M-QAM constellations and novel modulation techniques has been presented. QAMeleon's TPAs and ROADMs are being developed as main building blocks of an envisioned Colorless, Directionless, Contentionless and Gridless (CDCG) photonic technology.

\section{ACKNOWLEDGEMENTS}

This work has received funding from the European Union's Horizon 2020 research and innovation programme under grant agreement No. 780354 (QAMeleon).

\section{REFERENCES}

[1] Cisco white paper, "The Zettabyte Era: Trends and Analysis", 2016.

[2] OFC2017, “A Market Overview”, 2017.

[3] O. Riva et. al., in proc. OFC 2011.

[4] Víctor López et. al., J. Opt. Commun. Netw. 6, 476-485 (2014).

[5] Juan Pedro Fernandez-Palacios et. al., in proc. ECOC 2014, paper Mo.4.2.1.

[6] A. Napoli, et al., "Next generation elastic optical networks: The vision of the European research project IDEALIST," IEEE Commun. Mag., vol. 53, pp. 152-162, Feb. 2015.

[7] J. K. Fischer et al., "Bandwidth-Variable Transceivers based on Four-Dimensional Modulation Formats," J. Lightw. Technol., vol. 32, pp. 2886-2895, Aug. 2014.

[8] E. Agrell and M. Karlsson, "Power-Efficient Modulation Formats in Coherent Transmission Systems," J. Lightw. Technol., vol. 27, no. 22, pp. 5115-5126, Nov 2009. 
[9] E. Agrell, "Database of sphere packings," https://codes.se/packings/, accessed: 2018-01-31.

[10] Dd G. Forney et al., "Efficient Modulation for Band-Limited Channels,” IEEE J. Sel. Areas Commun., vol. 2, no. 5, pp. $632-647$, Sep 1984.

[11] G. Böcherer, F. Steiner, and P. Schulte, "Bandwidth efficient and rate-matched low-density parity-check coded modulation,” IEEE Trans. Commun.,vol. 63, no. 12, pp. 4651-4665, Dec. 2015.

[12] J. Renaudier, et al., "Comparison of Set-Partitioned Two-Polarization 16QAM Formats with PDM-QPSK and PDM8QAM for Optical Transmission Systems with Error-Correction Coding," in Proc. 38th Eur. Conf.Opt. Commun. (ECOC), Amsterdam, Netherlands, 2012, p. We.1.C.5.

[13] J. Conway and N. Sloane, "Fast quantizing and decoding and algorithms for lattice quantizers and codes," IEEE Trans. Inf. Theory, vol. 28, no. 2, pp. 227-232, Mar 1982.

[14] J. Cho, "Balancing Probabilistic Shaping and Forward Error Correction for Optimal System Performance," Proc. OFC 2018, paper M3C.2, San Diego, USA (2018).

[15] Wood M., "The Path to 100G Single Lambda in the Data Center - Part 6,", AFL, 10 May 2018, < https://www.aflglobal.com/AFL-Blog/May-2018/The-Path-to-100G-Single-Lambda-in-the-Data-Center.aspx> (21 January 2019).

[16] S. Dris, S. Alreesh, and A. Richter, "Blind Polarization Demultiplexing and Equalization of Probabilistically Shaped QAM," in Proc. Optical Fiber Communication Conference (OFC), paper W1D.2, San Diego, USA, Mar 2019.

[17] Snyder, Allan W., and John Love. Optical waveguide theory. Springer Science \& Business Media, 2012.

[18] N. Calabretta, "Photonic integrated WDM Cross-connects for Disaggregated Optical Metro-Access Networks," Asia Communications and Photonics Conference, 2017, paper S4J.1.

[19] N. Calabretta, W. Miao, K. Mekonnen, K. Prifti, and K. Williams, "Monolithically Integrated WDM Cross-Connect Switch for High-performance Optical Data Center Networks,"in Optical Fiber Communication Conference,OSA Technical Digest (online)(Optical Society of America, 2017), paper Tu3F.1.

[20] N. Calabretta, et al., "System Performance Assessment of a Monolithically Integrated WDM Cross-Connect Switch for Optical Data Centre Networks," ECOC, 2016, Paper W.2.F.3.

[21] IHS Infonetics Optical Network Hardware report.

[22] A. Deverajan et. al., in Proc. IEEE COMSNETS 2010

[23] Karsten Schuh, Fred Buchali, Wilfried Idler, Qian Hu, Wolfgang Templ, Anna Bielik, Lars Altenhain, Henning Langenhagen, Jörg Rupeter, Ulrich Dümler, Tobias Ellermeyer, Rolf Schmid, Michael Möller, “100 GSa/s BiCMOS DAC Supporting 400 Gb/s Dual Channel Transmission”, ECOC 2016. 